\title{
Risikogruppe „Alte“
}

atol blog.kulturwissenschaften.de/risikogruppe-alte/

\section{$\underline{\text { Fred Karl }}$}

\section{Vom Unsichtbarwerden der „neuen Alten“}

Sind sie vorbei, die Zeiten, als sich junge Studierende im Hörsaal die Plätze teilen mussten mit älteren und auch sehr alten Gasthörer*innen? Die Zeiten, als einige Oldies mit jungen Menschen, die ihre Enkelkinder hätten sein können, im Rockkonzert sich im Rhythmus bewegten und im gefühlvolleren oder gar besinnlicheren Teil des Events ihr Feuerzeug oder Handy zum Leuchten brachten? Zumindest fürs Erste scheint dies so, denn in der Corona-Krise ist die Ansteckungsgefahr für beide Gruppen hoch und gerade für die Älteren gefährlich.

Besondere Vorsicht wird sogenannten Risikogruppen anempfohlen. Die Gestalt der Risikogruppe existiert nicht erst seit Ulrich Becks „Risikogesellschaft“, die mit selbstgeschaffenen, vor allem ökologischen Risiken konfrontiert und sich dieser bewusst wird. Von Risikogruppen spricht man schon länger und in vielen gesellschaftlichen Funktionsbereichen, z. B. beim Arbeitsschutz und in der Verkehrssicherheit. Im Gesundheitsbereich werden Risikogruppen von der Epidemiologie identifiziert, um die Entstehung und Verbreitung von Krankheiten in der Bevölkerung zu messen.

Bei Infektionskrankheiten wie im Falle von COVID-19 wird benannt, welche Bevölkerungsteile besondere Risiken für eine Ansteckung, den Ausbruch einer Erkrankung und für einen schweren Krankheitsverlauf tragen. Es sind einmal jene mit Vorerkrankungen, nämlich Herz-Kreislauf-, chronischen Atemwegserkrankungen, Diabetes, Krebs, Adipositas und Bluthochdruck, bei denen das Risiko schwerer Verläufe erhöht ist. Zum anderen gilt auch das höhere Alter als Risikofaktor, weil im Alter das Immunsystem weniger gut reagiert. Mit diesen Definitionen gehören 30 bis 40 Prozent der Bevölkerung zur Corona-Risikogruppe. 


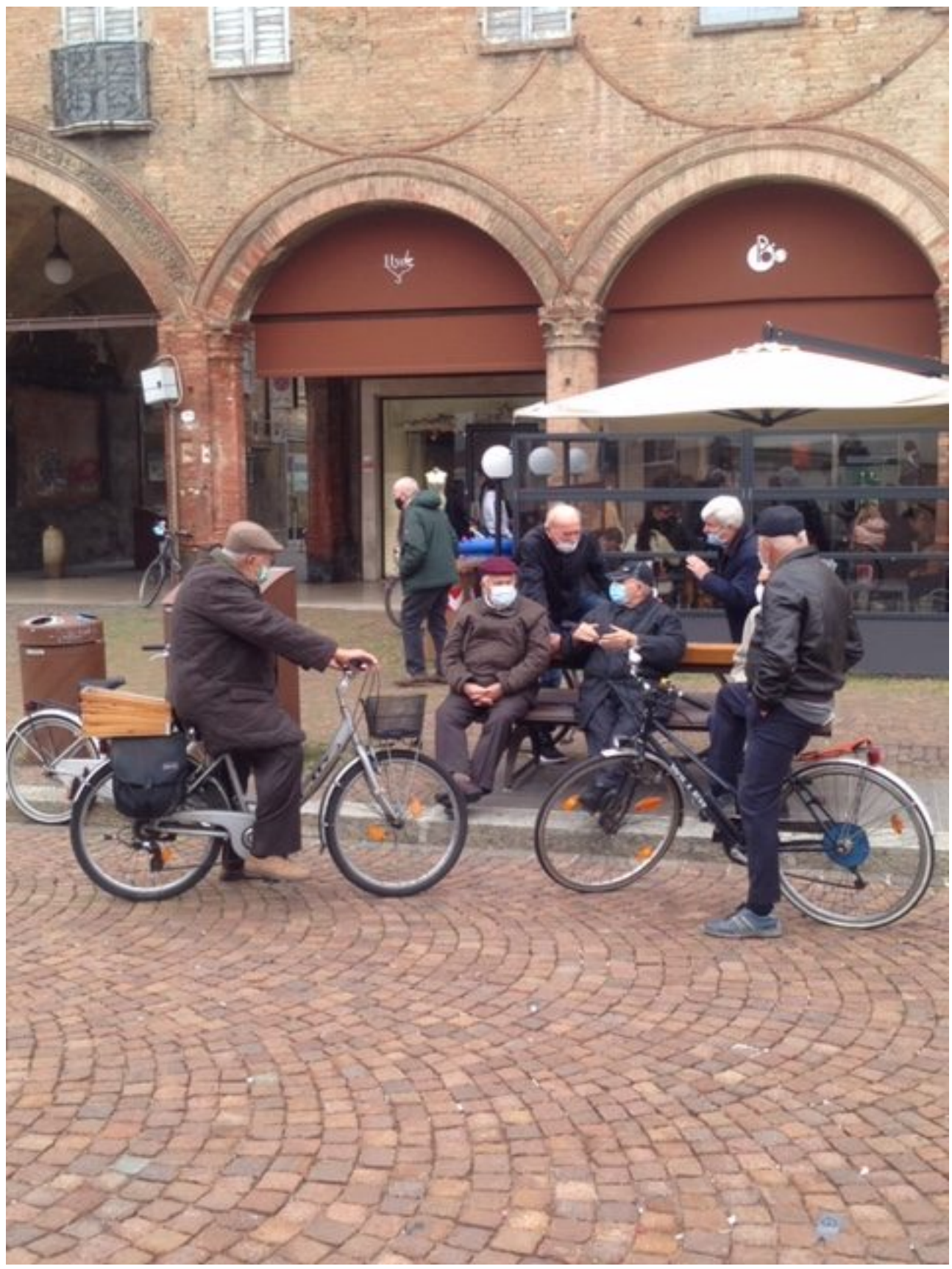

Abb. 1: Senioren in Carpi/Norditalien Ende Oktober 2020 (Foto: privat)

\section{Corona und die Alten}

Was COVID-19 betrifft, so sind zwar die Infektionszahlen bei den Jüngeren überdurchschnittlich, die Todesfälle jedoch sind zu 85 Prozent der Altersgruppe über 70 zuzuordnen. ${ }^{1}$ Während sich die Gruppen mit verschiedenen Vorerkrankungen figural kaum fassen lassen (von den Übergewichtigen einmal abgesehen), ermöglicht die Kategorie des höheren Alters ein deutlicheres Bild. Alte Menschen werden seit jeher gerne als gefährdet und hilfsbedürftig betrachtet. Sie sind es, von denen in der Pandemie ein Rückzug ins Häusliche erwartet wird. Alte Menschen in stationären Einrichtungen wurden von der Außenwelt isoliert, ihnen zeitweise der Besuch von Angehörigen verwehrt. 
In den USA verbreitete ein Radio-DJ die Botschaft, ältere Menschen sollten sich dem Coronavirus opfern, um die US-Wirtschaft zu retten. ${ }^{2}$ Auch der 69-jährige

Vizegouverneur von Texas, Dan Patrick, postulierte in einem Interview mit dem Fernsehsender Fox News ${ }^{3}$, dass die Großelterngeneration bereit sein solle, für die amerikanische Wirtschaft zu sterben, und nicht die Zukunft ihrer Enkel aufs Spiel zu setzen. Dieser Gefolgsmann des noch amtierenden Präsidenten Trump gibt als Angehöriger der Corona-Risikogruppe vor, seinen eigenen Tod in Kauf zu nehmen. Die viel jüngere Person, die auf dem folgenden Foto ein Schild mit der Aufschrift „Sacrifice the Weak“ hochhält, tritt nicht so vermeintlich selbstlos auf.

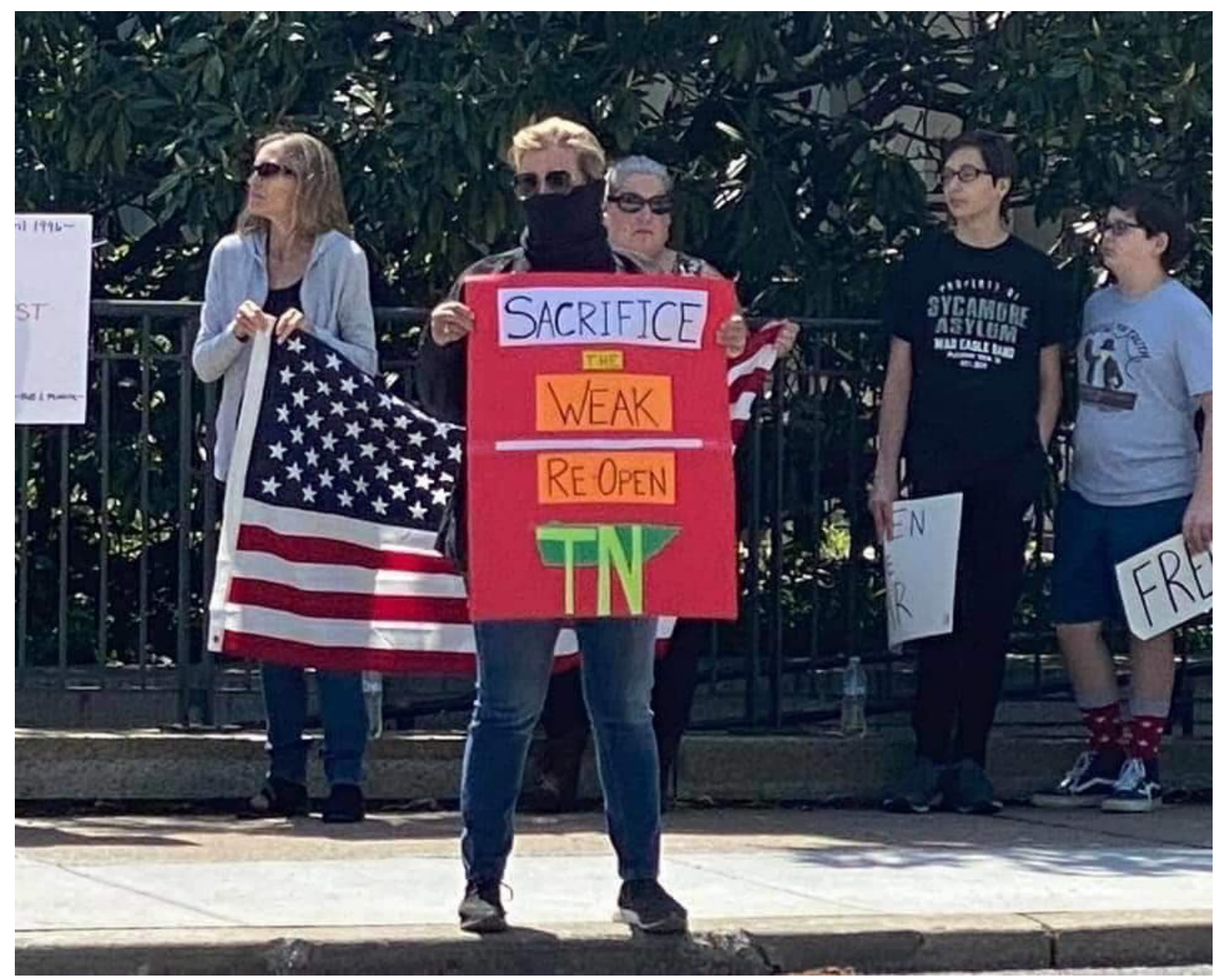

Abb. 2: Demonstranten in Nashville/Tennessee (https://pbs.twimg.com/media/EWNVSJhXkAEPYFo.jpg)

So wird deutlich, dass es im Diskurs um die Alten um mehr als um diese Gruppe geht: Nicht Solidarität mit gefährdeteren Menschen, sondern utilitaristische Postulate der gesellschaftlichen Nützlichkeit stehen im Vordergrund. Wird bald auch wieder gefragt, welches Leben überhaupt lebenswert ist?

In Deutschland sorgte der Tübinger Oberbürgermeister Boris Palmer mit folgender Äußerung - die er später zurück nahm - für bundesweites Aufsehen: „Wenn Sie die Todeszahlen durch Corona anschauen, dann ist es bei vielen so, dass viele Menschen über 80 sterben - und wir wissen, über 80 sterben die meisten irgendwann. (...) Ich sage es Ihnen mal ganz brutal: Wir retten in Deutschland möglicherweise Menschen, 
die in einem halben Jahr sowieso tot wären - aufgrund ihres Alters und ihrer Vorerkrankungen."4 Das Medianalter der COVID-Sterbefälle liegt in Deutschland tatsächlich bei 81 bzw. 82 Jahren. Aber: Die Menschen, die es geschafft haben, so alt zu werden, wären (trotz eventueller Vorerkrankungen) nicht in einem halben Jahr verstorben, sondern haben noch einige Jahre vor sich: So weisen die KohortenSterbetafeln des Statistischen Bundesamtes für die heute 80-Jährigen eine restliche durchschnittliche Lebenserwartung von weiteren acht Jahren für die Männer und 9,5 Jahren für die Frauen aus. Gegenüber dem Altersschicksal früherer Generationen sind dies nicht zu unterschätzende „gewonnene Jahre“. Manche schaffen es über die 90, oder gelangen in die nun schon mehrere Tausende zählende Gruppe der Hundertjährigen.

Dessen ungeachtet muss jedoch gesagt werden, dass die Argumentationsfigur, alte Leute hätten sowieso nicht mehr lange zu leben, auf den eigentlich kritischen Punkt gar nicht eingeht. Dieser liegt im Zusammenhang von Alter, Sozialschicht, Morbidität und Mortalität. So sind Vorerkrankungen keineswegs sozial gleich verteilt, was sozialepidemiologische Untersuchungen beispielsweise zur Prävalenz von HerzKreislauf-Erkrankungen gezeigt haben. Personen mit niedriger Schichtherkunft und Bildungsqualifikation sind öfter von solchen gesundheitlichen Einschränkungen betroffen als Personen mit gutem Schul- und Berufsabschluss und höherem Einkommen. Und erst recht decken sozioökonomische Analysen der Todeszahlen auf, dass sozial Benachteiligte eine weitaus geringere Lebenszeit haben als privilegiertere Schichten. Man muss sich konkret nur die Restlebenserwartung. ${ }^{5}$

Die Sozialfigur der „neuen bzw.jungen Alten“

,Das Alter ist nicht homogen. Die Heterogenität des Alters wird sozialwissenschaftlich immer wieder belegt, nicht nur anhand sozioökonomischer Parameter, sondern auch hinsichtlich in Jahrzehnten kumulierter Lebenserfahrungen, Lebensstile und Verhaltensweisen. Sie zeigt sich auch in der Pluralisierung der Altersbilder.

Das lange Zeit typische Bild der gebeugten „Betagten“ mit Gehstock, noch auf Straßenverkehrsschildern in Ländern des früheren British Empire und gelegentlich in Piktogrammen zu sehen, wurde - vor Corona - abgelöst durch die neutralere Bezeichnung „Senioren“ und „Seniorinnen“ und deutlicher noch durch die Sozialfigur der „neuen Alten“.

Zunächst hat das Auftauchen neuer Altersbilder ökonomische Gründe. Aufgrund der Situation auf dem Arbeitsmarkt ermöglichte in Westdeutschland in den 1980er Jahren das Vorruhestandsgesetz einen Übergang in den Ruhestand mit 59 Jahren, in Ostdeutschland wurden nach der Wende in den 1990er Jahren ältere Beschäftigte schon mit 55 Jahren im Zuge der Abwicklung ganzer Wirtschaftsbranchen in die Frührente geschickt. Nun war die Rede vom „Unruhestand“ und von „aktiven Alten“. 
Die Wissenschaft vom Altern, die Gerontologie, hebt seitdem gerne Kompetenzen und Potenziale im Alter hervor. Problemgruppen unter älteren Menschen werden weniger thematisiert, um nicht die in den Köpfen weiterhin vorhandenen negativen Altersstereotype zu stärken. Stattdessen wird ein ,erfolgreiches` Leben im Alter idealisiert, wobei man Gefahr läuft, die nicht leistungsfähigen Alten zu diskriminieren, wenn sie dem neuen Leitbild nicht gerecht werden können. Empirische Studien dieser Zeit, wie die zu Beginn der 1990er Jahre durchgeführte Lebensstilanalyse von Infratest/Sinus/Becker ${ }^{6}$, fandenunter den befragten $55^{-}$bis $70-$ Jährigen eine Mehrheit mit eher „pflichtbewusst-häuslichen“, „sicherheits- und gemeinschaftsorientierten“ oder „resignierten“ Einstellungen. Die Minderheit, ein Viertel, machten die aktiveren, besser gebildeten, gesünderen, mobileren und wohlhabenderen Befragten aus. Sie wurden als die „neuen Alten“ in den Vordergrund gestellt. Diese Begrifflichkeit knüpfte an die wenige Jahre zuvor entwickelte Sozialfigur der „neuen Alten“ an (schon 1985 war ein Reader mit Rundfunkvorträgen über die „neuen Alten“ erschienen, 1,988 befasste sich die Jahrestagung der Deutschen Gesellschaft für Gerontologie mit ihnen). Später wurde diese Gruppe als ,junge Alte"7 etikettiert.

Nachdem auch in das Selbstverständnis vieler Älterwerdender die freundlichere Beschreibung als „neue“ bzw. ,junge

Alte"einsickerte, erkannte die Wirtschaft in ihnen eine marketingrelevante Zielgruppe. In der Marketingsprache kam die Adressierung als „Best Ager“ auf. Auch der Bildungsbereich stellte sich mit altersspezifischen Kursen an Volkshochschulen und „Universitäten des Dritten Lebensalters" auf sie ein.

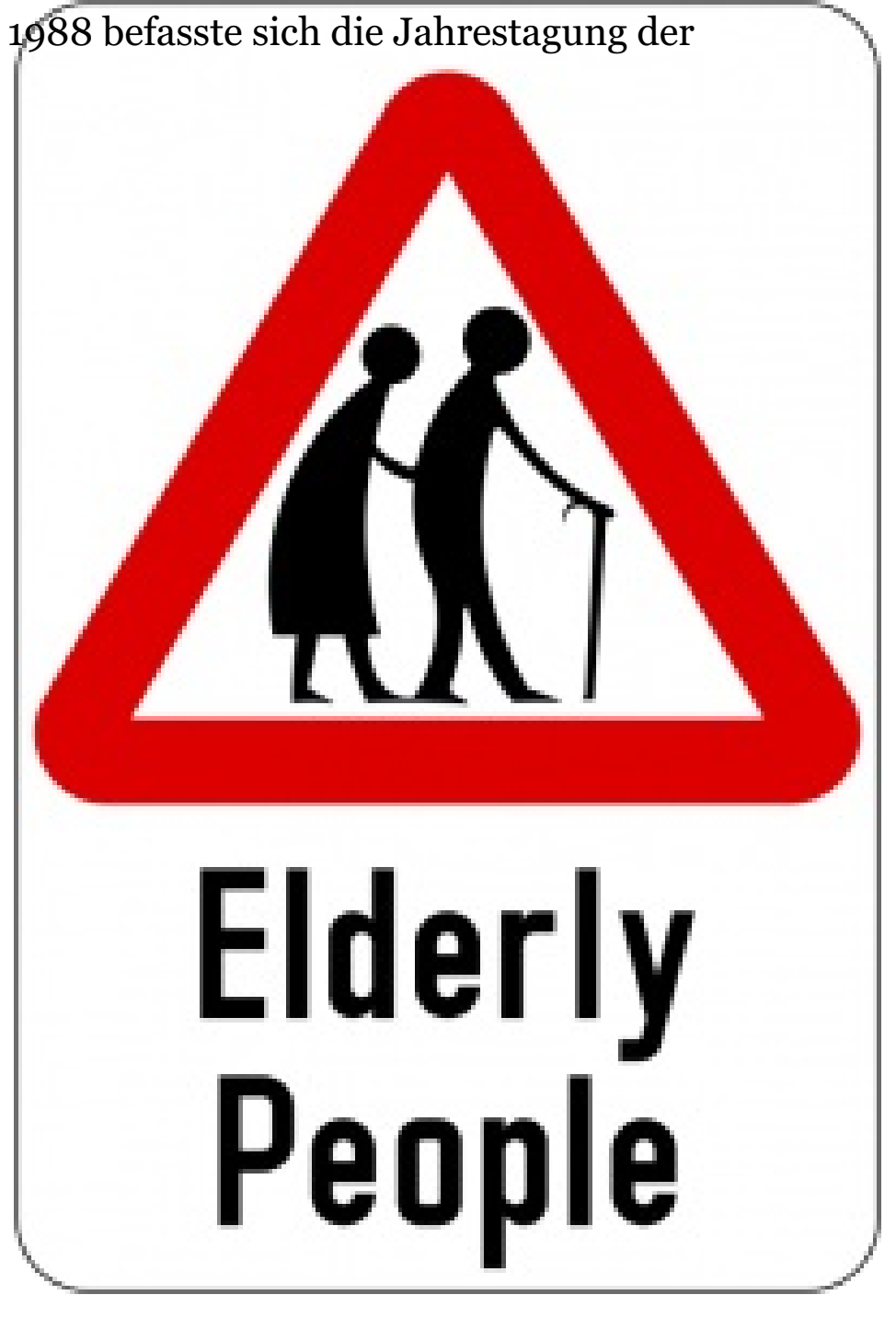

Abb. 3: Verkehrsschild in Großbritannien (Public domain, via Wikimedia Commons) 


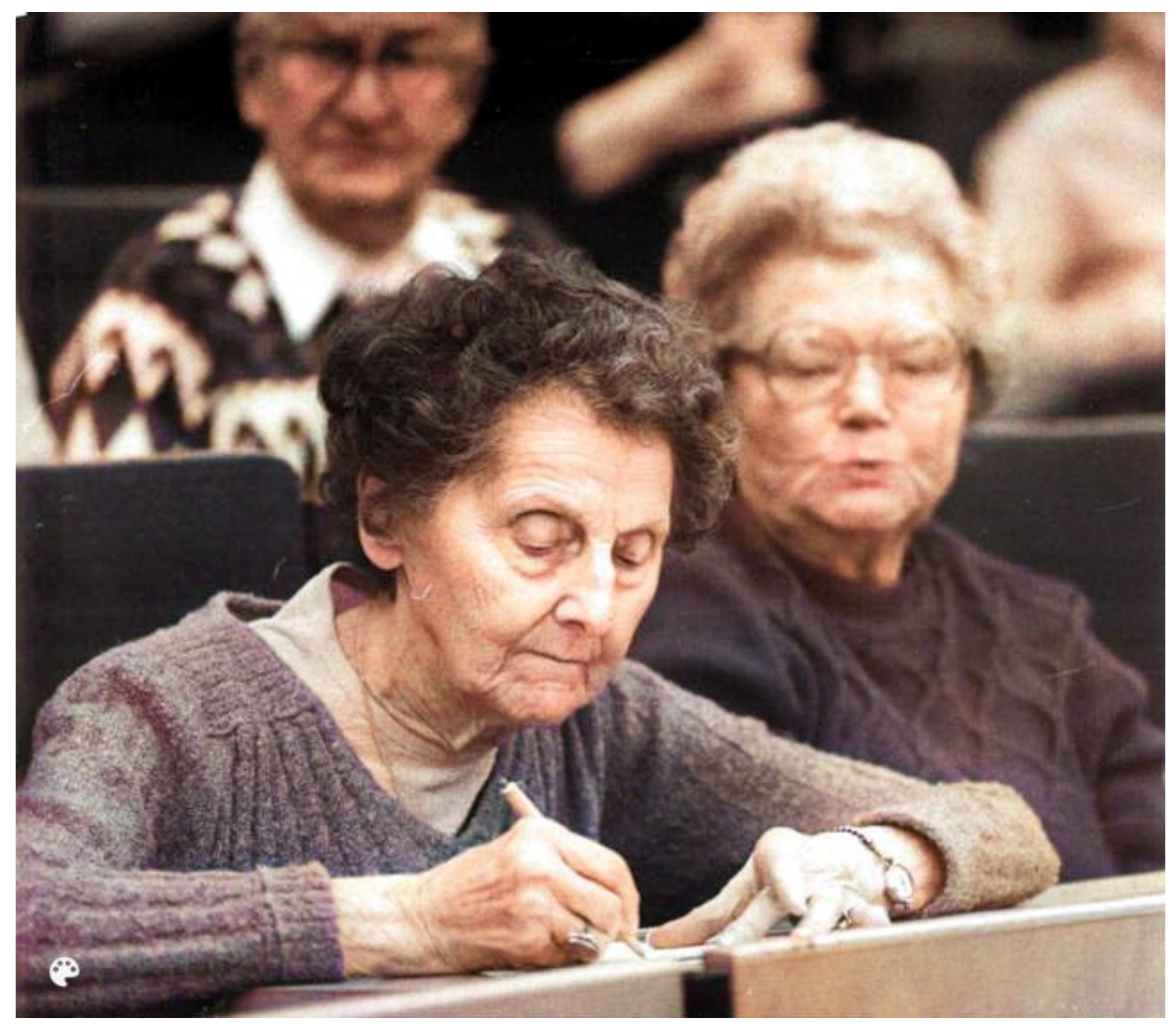

Abb. 4: „Seniorenstudenten“ an der Universität Leipzig in den 1990er Jahren (Bundesarchiv, Bild 183-1986-0109-010 / https://commons.wikimedia.org/w/index.php?curid=88741910)

Im altenpolitischen Bereich vollzog sich seitdem ein bewusst gesteuerter Paradigmenwechsel vom umsorgten Alter hin zur Aktivierung für ehrenamtliche Tätigkeiten. Ausdruck dieser Neuorientierung ist die Etablierung von Seniorenbüros als Anlauf- und Koordinierungsstellen für Senioren-Engagement, um deutlich zu machen, dass es sich bei älteren Menschen um „aktive, verantwortungsbewusste, leistungswillige und leistungsfähige Menschen “ 8 handelt. Dabei entdeckte die Politik das „Erfahrungswissen Älterer“. Ihr bürgerschaftliches und ehrenamtliches Engagement wurde von Bundes- und Landesministerien mit Modellprogrammen gefördert, ihr damit geleisteter wohlfahrtsstaatlicher Beitrag ökonomisch berechnet. Schon wurde von anderer Seite kritisch nachgefragt: Waren die „neuen Alten“ somit in gewisser Weise auch „Retter des Sozialen“ oder gar Lückenbüßer für fehlende Stellen in der Sozialen Arbeit?9

Es entstand mit diesen Programmen auch eine gestiegene Erwartung an die Alternden, ,aktiv“ zu bleiben und sich einzubringen. Manche zeigten sogar Spuren der „Überaktivität“, wie der Heidelberger Alterspsychologe Hans-Werner Wahl feststellt. Zusätzlich hinterfragt der Kasseler Mediziner und Psychotherapeut 
Hartmut Radebold, ob die anhaltende Leistungsorientierung der Generation der Kriegs- und Nachkriegskinder auch daher rühre, dass diese mit unhinterfragtem Aktivismus ihre unbearbeiteten Traumata überspielten.

\section{Die (neuen) Alten in der Corona-Pandemie}

Im Zuge der Corona-Pandemie wird die Sozialfigur der ,jungen Alten“ jedoch dramatisch verunsichert. Mit einem Mal finden sich die vormaligen Träger von Ressourcen, Potentialen und Kompetenzen in einer Risikogruppe wieder. Deutet die überwiegende Zustimmung zu den Corona-Schutzmaßnahmen unter den Älteren darauf hin, dass sich der schon immer präsente Habitus der oben erwähnten mehrheitlichen Häuslich-Sicherheitsorientierten gegenüber den mobilen, außerfamiliär Orientierten durchgesetzt hat? Oder haben sich letztere derzeit nur unsichtbar gemacht - abgesehen von unerschrockenen Personen wie dem 85jährigen Dieter Hallervorden, der sich für die Offenhaltung kultureller Einrichtungen einsetzt? ${ }^{10}$

Nun, zum einen sind die ersten Protagonisten der „neuen Alten“ inzwischen im sehr hohen Alter angelangt. ${ }^{11}$ Zum anderen wachsen in die Gruppen der aktiven jungen Alten weitere Akteure nach, und zwar aus den Folgegenerationen der altgewordenen 68er und in Zukunft aus den Babyboomer-Jahrgängen. Wie bei aufeinander folgenden Jugendkulturen verändert auch hier der Nachwuchs das Erscheinungsbild und die Performanz der Altengenerationen, so dass die Sozialfigur der ,jungen“, „aktiven“ und „neuen Alten“ von den nachrückenden, ins Alter kommenden Männern und Frauen weitere Akzentuierungen erfahren dürfte. Dies zeigt sich gegenwärtig auch darin, dass die immer wieder neuen Alten nun auch die anfangs den Digital Natives vorbehaltene virtuelle Welt betreten, wie die wachsende Szene der „Silversurfer“ und Online-Gruppen wie „Digitalisierung und Engagement“ erkennen lassen. ${ }^{12}$

Es bleibt abzuwarten, ob und wie die Sozialfigur der „aktiven bzw. neuen Alten“ mit der Überwindung der Pandemie und der Kontaktbeschränkungen wieder offline in der Öffentlichkeit Präsenz zeigt und welche Wahrnehmung sie dann erfährt. Allerdings dürfte in Nach-Corona-Zeiten das überwältigende Thema des Klimawandels in den Vordergrund rücken.

Auch wenn gegenwärtig im Verlaufe einer Virus-Pandemie der räumliche Abstand geboten ist, wird zur Lösung der ökologischen und ökonomischen Zukunftsfragen ein Zusammenrücken - und sei dies zunächst virtuell - von Alt und Jung erforderlich sein. Obwohl dies bei manchen noch nicht angekommen zu sein scheint: Die drohende Klimakatastrophe macht alle Menschen zu Mitgliedern einer weltweiten Risikogruppe.

Beitrag ist lizensiert unter einer CC BY-SA 4.0 Lizenz. 


\section{References}

1. Siehe die Lageberichte des Robert Koch Instituts: https://www.rki.de/DE/Content/InfAZ/N/Neuartiges Coronavirus/Fallzahlen $\underline{\mathrm{html}}$

2. https://metro.co.uk/2020/03/25/dj-says-elderly-people-sacrifice-coronavirussave-economy-12454758/

3. https://eu.usatoday.com/story/news/nation/2020/03/24/covid-19-texasofficial-suggests-elderly-willing-die-economy/2905990001/

4. https://www.sat1.de/tv/fruehstuecksfernsehen/video/202082oberbuergermeister-boris-palmer-spricht-ueber-die-deutsche-wirtschaft-clip

5. Ich danke IFSOZ für die Anregung zu diesem anschaulichen Bild.

6. Infratest/Sinus/Becker (1991): Die Älteren. Zur Lebenssituation der 557ojährigen. Bonn: Friedrich Ebert Stiftung.

7. Silke van Dyk und Stephan Lessenich (2009): Die jungen Alten. Analysen zu einer neuen Sozialfigur. Frankfurt a.M. und New York: Campus.

8. Bundesministerium für Familie und Senioren (Hrsg.), (1994): Materialien zum Modellprogramm Seniorenbüros. Band 1. Bonn: BMFuS, S. 13

9. Kirsten Aner, Fred Karl und Leopold Rosenmayr (2007): Die neuen Alten Retter des Sozialen? Wiesbaden: VS. https://doi.org/10.1007/978-3-53190472-6.

10. Ich danke Sebastian Moser für diesen Hinweis und für die inhaltliche Begleitung bei der Überarbeitung dieses Artikels.

11. Fred Karl (Hrsg.), (2012): Das Altern der „neuen“ Alten. Eine Generation im Strukturwandel des Alters. Berlin: LIT.

12. Ich danke der KWI-Redaktion für hilfreiche Überarbeitungsvorschläge.

SUGGESTED CITATION: Karl, Fred: Risikogruppe „Alte“. Vom Unsichtbarwerden der „neuen Alten“, in: KWI-BLOG, [https://blog.kulturwissenschaften.de/risikogruppe-alte/], 21.12.2020

DOI: https://doi.org/10.37189/kwi-blog/20201221-0830 


\section{DuEPublico}

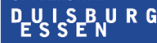

Duisburg-Essen Publications online

offen im Denken

Ub $\begin{aligned} & \text { universitäts } \\ & \text { bibliothek }\end{aligned}$

Dieser Text wird über DuEPublico, dem Dokumenten- und Publikationsserver der Universität Duisburg-Essen, zur Verfügung gestellt. Die hier veröffentlichte Version der EPublikation kann von einer eventuell ebenfalls veröffentlichten Verlagsversion abweichen.

DOI: $\quad 10.37189 / \mathrm{kwi}-\mathrm{blog} / 20201221-0830$

URN: urn:nbn:de:hbz:464-20201221-105802-1 